\title{
THE PULSATION PROPERTIES OF RED VARIABLES
}

\author{
M.W. Feast \\ South African Astronomical Observatory, Cape.
}

\section{ABSTRACT}

Recently derived bolometric absolute magnitudes are used to investigate the pulsational properties of some classes of red variables. The Mira variables with periods over 200 days have pulsation constants appropriate to overtone pulsators but the shorter period 135 day Miras seem to be hotter and less luminous and may be fundamental pulsators. The large amplitude Shapley-Nail variables in the SMC are probably fundamental pulsators with masses in the 5-10 solar range. Bright supergiant red variables in the LMC show evidence of the period-luminosity relation expected if they are in the early stages of core helium burning.

At the present time the nature of the pulsations in red variables, particularly the Mira type variables, is a matter of quite heated debate. Wood concluded some while ago from pulsational calculations on relevant stellar models that the bulk of Mira variables must be pulsating in the first overtone. Associated with this view was the idea that the more violent fundamental pulsations might be a suitable mechanism leading to extensive mass ejection and the production of objects which could be identified with the symbiotic stars. More recently most of the evidence has favoured the view that symbiotic stars are interacting binaries, a cool giant (in some cases a Mira variable) and a much denser close companion. What becomes of the fundamental pulsators remains therefore something of a mystery. The introduction of infrared Fourier transform spectrometers (for instance the work at Kitt Peak by Hal1, Hinkle and Ridgway) (cf. IAU Colloquium 46 in press) has opened up the possibilities of greatly improved studies of the atmospheric kinematics of Mira variables during their pulsation cycle. Hill and Willson (cf. Ap. J. 229, 1029, 1979) have made rather extensive numerical investigations of hydrodynamical 
models (including shock waves) for Mira variable atmospheres. They conclude that their results are much better satisfied by assuming fundamental mode pulsations ( $\sim 0.1$ ) than first overtone pulsations $(Q \sim 0.05)$. I expect that $\mathrm{Dr}$ Wood in his talk will pay some attention to this controversial matter which clearly needs to be resolved if we are to understand the nature of the instabilities in red giant atmospheres. What I would like to do is to review the present position regarding the absolute magnitudes, particularly the bolometric absolute magnitudes, of the Mira variables and to see what this has to tell us about this problem. In addition $I$ want to make some comments on supergiant red variables.

An extensive body of infrared broad band ( $J, H, K, L$ that is $1.2,1.6,2.2,3.4 \mu$ ) photometry of red variables has been recently published by a group at SAAO. There are about 3000 sets of observations of which about 2000 are of 223 Mira variables with Me spectra. Since most of the energy of late type variables is radiated in this spectral range the results can be used to derive quite satisfactory bolometric magnitudes and the colours can be at least approximately calibrated in terms of temperature.

A plot of the mean $(\mathrm{J}-\mathrm{K})$ colour against period for the Mira variables shows quite a good correlation. The temperature of Mira variables near mean light drops as the period increases. Whilst it is useful to have quantitative information on this point, perhaps the most interesting feature of these results is that the short period Miras with periods between 90 and 150 days continue the general relation to these periods. They are in the mean hotter than the longer period stars. These stars are known to form a puzzlingly anomalous group as we sha1l see later.

Mean absolute bolometric magnitudes have been derived in the following ways using the new infrared photometry:

(1) Statistical parallaxes (by Robertson) for 179 stars with known proper motions for which we have photometry. This excludes 93 northern variables with proper motions which are not observable from Sutherland. (2) Visual absolute magnitudes from statistical parallaxes from the full set of proper motions, with mean bolometric corrections derived from the infrared photometry. (3) Individual distances for Mira variables in globular clusters, visual binaries, Eggen groups, with a measured parallax (o-Ceti) or a distance from interstellar line strengths ( $X$ Mon). There is appreciable scatter in these absolute magnitudes. A considerable part of this is probably observational. In the mean the Miras show a slow rise in brightness with period, from Mbol $\sim-4.1$ at 200 days to Mbo1 -4.6 at 400 days. Confirmation of these figures is provided by some work by Glass on a few Miras discovered by 
Lloyd Evans in the Large Magellanic Cloud. These lie in the range $M_{\mathrm{bo}}-4.2$ to -5.1 . It seems very likely that intensive work in the Magellanic Clouds will provide the best periodluminosity relation and that it will be especially valuable in establishing the size of the intrinsic scatter in this relation. This latter point is of course vital for assessing the value of Miras as distance indicators. The above discussion leaves out the short period ( 135 day) Miras and the statistical parallaxes suggest these stars to be distinctly fainter than the rest $\left(M_{\text {bol }} \sim-2.5\right)$.

For many purposes it is simplest to work with the absolute magnitude at $\mathrm{K}(2.2 \mu)$. As with the bolometric magnitude this shows a slow rise in brightness from 200 to 400 days with the shorter period stars fainter. A useful comparison of these $K$ absolute magnitudes can be made with recent work by Glass on Miras in the Baade windows towards the galactic centre. We expect most of these stars to be concentrated near the centre so that their range in distance from us is smal1. The $\mathrm{K}$ apparent magnitudes of these stars show a slow rise in brightness from 200 to 400 days but with the few shorter period variables measured distinctly fainter. This therefore agrees with our absolute magnitude calibration.

Our results on the bolometric absolute magnitudes and colours can be used to look at the pulsational properties of Miras. Arranging the $P \sqrt{ } \rho$ relation as a function of period, mass, luminosity and temperature in the usual way we find that for the bulk of the Miras (200 - 400 day periods) the pulsation constant $Q_{a}$ is about $0.06 \mathrm{M}$ where $M$ is the mass in solar units. $M$ is in all probability not greatly different from unity. These results would suggest that the bulk of the Miras are overtone pulsators for which Wood and others predice $\mathrm{Q} \sim 0.05$. Applying the same procedure to the $\sim 135$ day Miras which we found to be hotter and less luminous, we get $\mathrm{Q}_{\mathrm{b}} \sim 0.15 \mathrm{~V} \mathrm{M}$. With $\mathrm{M}$ near unity this suggests fundamental mode pulsation for these stars. The galactic kinematics of Miras shows a gradual change of population type as the period decreases from 400 to 200 days with the 90 - 150 day variables being kinematically associated with "main stream" miras of $\mathrm{P} \approx 330$ days. If this is taken to indicate equal masses in the 330 day and 135 day groups then $Q_{b} / Q_{a} \sim 2.5$ which is about the ratio expected for fundamental to first overtone pulsation. These results are quite surprising because they suggest that red giants of a given mass pulsate in the fundamental when they are relatively small and hot and in the first overtone when they are bigger and cooler. This is quite the opposite of theoretical prediction. It is clear that every effort should be made to improve the observational basis of this discussion. If the results are substantiated they appear to suggest either (1) the theoretical prediction is wrong or (2) cool fundamental 
pulsators are removed from the scene by catastrophic instabilities or (3) the 135 day Miras have very low masses perhaps due to having undergone exceptional mass loss.

I have already mentioned the Mira variables in the LMC and I want now to discuss briefly two other groups of Magellanic Cloud variables. Firstly, the Shapley-Nail variables in the SMC. These are long period variables, of ten with periods in the range $500-800$ days and with large amplitudes. These stars are often compared with galactic Miras though their visual absolute magnitudes are considerably brighter. Recently Glass has observed six or seven of these in the infrared (MNRAS 186, 317, 1979). Their bolometric absolute magnitudes are in the range -6.4 to -7.4 , considerably brighter than one would expect if one extrapolated the galactic Mira luminosities to these periods. Furthermore, despite their long periods, their colours indicate that they are as hot as the shorter period Miras. The likelihood seems to be that these stars are considerably more massive than normal Miras, possibly having masses in the 5 - 10 solar mass range. Although more work needs to be done on this problem the indications are that variables of this type are more prevalent in the SMC than in the LMC or the Galaxy. Should this prove to be the case it would suggest that the temperature at which instability sets in, in this mass range, is a function of chemical composition.

Finally, a number of bright supergiant red variables have long been recognized in the LMC. Glass has published infrared photometry for five of these. The bolometric absolute magnitudes lie close to a period-luminosity relation which can be predicted (cf. Stothers and Leung AA 10, 290, 1971) using a theoretical pulsation constant $Q=0.06$ (fundamental mode) for massive red stars, and masses derived from a theoretical massluminosity relation for massive stars in the early stages of core helium burning and temperatures near $3000^{\circ} \mathrm{K}$ which are in moderately good agreement with the observed colours. The masses of the stars involved lie in the range $20-35$ solar masses. These results are very encouraging and one might hope with more extensive observations to obtain a much improved period-luminosity relation for red supergiants. Obviously this would be of value not only in studies of stellar instabilities but also for distance scale problems. 